\title{
Estudio de la satisfacción percibida por los estudiantes de la UNED con su vida universitaria
}

\section{Study of UNED student satisfaction with their university life}

\author{
Margarita González-Peiteado \\ Universidad Nacional de Educación a Distancia (España) \\ Margarita Pino-Juste \\ Universidad de Vigo (España) \\ María Penado Abilleira \\ Universidad Nacional de Educación a Distancia (España)
}

\section{Resumen}

La evaluación de los planes de calidad universitarios se ven optimizados cuando éstos incorporan opiniones e indicadores de satisfacción estudiantil. En consecuencia, partiendo del modelo propuesto por Kember y Leung (2005), el objetivo de esta investigación es analizar la satisfacción con la vida universitaria a través de encuestas online al alumnado que cursa estudios en la Universidad Nacional de Educación a Distancia (UNED, España). Los datos obtenidos se tabulan originando un modelo que nos permite identificar aquellos elementos que influyen de manera significativa en la satisfacción del alumnado en relación a cuatro dimensiones: docencia, contenidos, comunicación y capacidades. Para ello participaron 60 estudiantes de diferentes titulaciones correspondientes a cinco ámbitos académicos con edades comprendidas entre 24 y 50 años. Con la intención de conocer e interpretar una realidad y de abrir un debate sobre la optimización de la calidad educativa, se ha optado por un diseño no experimental de carácter exploratorio, descriptivo y transversal. Se realizaron estudios de consistencia interna realizando un análisis factorial exploratorio y coeficiente Alfa de Cronbach $(\alpha=0.96)$. Se pone de manifiesto el alto nivel de satisfacción de los estudiantes con la experiencia universitaria, evidenciando un elevado grado de bienestar en las variables analizadas. Los resultados revelaron la existencia de diferencias significativas debidas a la edad y al ámbito académico. La revisión de las variables implicadas en el constructo satisfacción con la vida universitaria nos ha permitido señalar la necesidad de optimizar estrategias y recursos relacionados fundamentalmente con el factor docencia para la mejora de la calidad universitaria.

Palabras clave: evaluación; satisfacción; estudiantes; educación superior; comunicación. 


\begin{abstract}
The evaluation of university quality plans are optimized when these incorporate views and indicators of student satisfaction. As a result, based on the model proposed by Kember and Leung (2005), the objective of this research is to analyze satisfaction with University life through online surveys to students who studied at the Universidad Nacional de Educación a Distancia (UNED, Spain). The resulting data are tabulated a model that allows us to identify those elements that have a significant influence on student satisfaction in relation to four dimensions: teaching, content, communication and capabilities. This was attended by 60 students from different degree programs corresponding to five academic areas aged between 24 and 50 years. Intending to understand and interpret a reality and to open a debate on optimizing the quality of education, we have opted for a non-experimental design of exploratory, descriptive and transversal character. Studies of structure and internal consistency were conducted by performing an exploratory factor analysis and Cronbach's alpha coefficient $(\alpha=0.96)$. It shows the high level of student satisfaction with the University experience, demonstrating a high degree of comfort in the variables analyzed. The results revealed significant differences due to age and the academic field. The review of the variables involved in the construct University life satisfaction has allowed us to point out the need to optimize strategies and resources fundamentally related to the teaching factor in order to improve University quality.
\end{abstract}

Keywords: evaluation; satisfaction; students; higher education; communication.

El alumnado universitario como actor beneficiario de la formación académica constituye un indicador fundamental para el estudio de la calidad de las instituciones. Un alumnado satisfecho con la formación recibida identifica y confirma la calidad de la misma, lo que redunda en una mayor motivación e implicación, pero también en el desarrollo de un sentimiento de pertenencia y deseos de intervenir en los distintos cauces de participación favoreciendo una difusión positiva de la actividad académica y la permanencia en las aulas (Sánchez-López, 2012). La satisfacción estudiantil ha sido concebida como el grado de congruencia entre las expectativas previas de los estudiantes y los resultados obtenidos, con respecto a la experiencia de aprender (Allen, Omori, Burrell, Mabry y Timmerman, 2013; Moore y Shelton, 2014). Para Gento Palacios y Vivas García (2003) la satisfacción es el resultado de un proceso valorativo. En consecuencia, cuando un individuo manifiesta satisfacción o rechazo hacia algo, está emitiendo un juicio de valor a partir de un proceso de evaluación. Los citados autores profundizan en la definición considerando la satisfacción estudiantil como la apreciación favorable que hacen los estudiantes de los resultados y experiencias asociadas con su educación, en función de la atención a sus propias necesidades y al logro de sus expectativas. 


\section{ALGUNOS ESTUDIOS RELEVANTES}

Estudios llevados a cabo sostienen que los estudiantes que presentan un alto grado de satisfacción con la universidad se caracterizan por progresar adecuadamente en sus metas académicas, poseer elevadas creencias acerca de sus capacidades para lograr un buen rendimiento en las tareas, desarrollar expectativas positivas respecto a la vida universitaria constatando la existencia de apoyo social para alcanzar sus objetivos educativos (Medrano y Pérez, 2010). Otros autores coinciden en vincular la satisfacción con el bienestar psicológico, con una visión eudemónica del bienestar unida a la autorrealización personal a partir de la consecución de objetivos (Fernández-Abascal, et al., 2011).

Para Pérez Juste (2000) la satisfacción hace referencia a la adecuada respuesta a las expectativas, intereses, necesidades y demandas de los destinatarios. "En el caso particular del ámbito educativo, los destinatarios son los alumnos, y su satisfacción se relaciona con la manera en que el proceso educativo y la institución misma atienden las expectativas, intereses y necesidades de este grupo particular" (Gento Palacios y Vivas García, 2003, p. 19).

Por ello, se concibe la satisfacción como una dimensión positiva del bienestar psicológico personal, el resultado de la comparación entre las aspiraciones y los logros. Dicho análisis puede ser realizado de forma holística o fragmentada, teniendo en cuenta aspectos de nuestra vida como vida académica, familia o trabajo.

La literatura existente sostiene que la calidad de vida de los estudiantes universitarios se focaliza en su bienestar subjetivo. Sirgy, Grezeskowiak y Rahtz, (2007) presentan un modelo que acomete la medición de la calidad de vida universitaria mediante el estudio de la satisfacción de los estudiantes. Este modelo es utilizado por autores como Yu y Lee (2008) o más recientemente El Hassan (2011), que determinan cómo la satisfacción con los diferentes componentes de la universidad configura la calidad de vida universitaria. Siguiendo con la revisión de bibliografía llevada a cabo en trabajos del contexto internacional comprobamos que ya existen algunos trabajos sobre la satisfacción de los estudiantes matriculados en instituciones de educación a distancia (Joo, Lim y Park, 2011; Keengwe, Diteeyont y Lawson-Body, 2012; Lee, Srinivasan, Trail, Lewis y López, 2011; Özkök, Walker y Büyüköztürk, 2009) y en otras instituciones educativas (Moosmayer y Siems, 2012). Algunos de estos trabajos orientan la investigación en la relación entre la calidad de vida de los estudiantes y variables como la personalidad, la salud y el entorno (Váez, Kristenson y Laflamme, 2004; Chow, 2005). Otros se refieren a aspectos como docencia, ámbito de estudio, comunicación, sexo (Blázquez, Chamizo, Cano y Gutiérrez, 2013; Solinas, Masia, Maida y Muresu, 2012).

No obstante, como señala Zambrano (2016), al iniciar el proceso de evaluación de la satisfacción del alumnado se observa que la mayoría de las investigaciones publicadas han sido hechas entre poblaciones angloparlantes; hay muchos factores relacionados con la satisfacción estudiantil, de los cuales algunos términos se 
refieren a una misma variable (e.g., interactividad e interacción estudiantesestudiantes) y diferentes variables son denominadas con un mismo término (e.g., docente, instructor, o facilitador); a lo que se añade la existencia de instrumentos para diferentes factores que se miden con diversas escalas.

En nuestro entorno, Fernández-Pascual, Ferrer-Cascales y Reig-Ferrer (2013) valoran el grado de satisfacción de los estudiantes con la formación recibida en un entorno virtual. A su vez, Blázquez Resino, Chamizo González, Cano Montero y Gutiérrez Broncano (2013) identifican los principales indicadores de la satisfacción del estudiante con la universidad. Flores Alarcia (2012) se cuestiona si cambian las metodologías docentes según el grado de presencialidad de las asignaturas. Sin embargo, no existen estudios recientes en nuestro contexto sobre el grado de satisfacción del universitario en enseñanzas a distancia.

La educación a distancia fue evolucionando a lo largo del siglo XX como un acceso alternativo a la formación en la que no existen las rigideces espaciotemporales propias de la docencia convencional (García Aretio, 2001, 2016) dirigida a aquellas personas que, bien por su situación geográfica (alumnos en zonas rurales), sus condiciones de trabajo (personas con poco tiempo para atender una enseñanza reglada), sus condiciones físicas (personas con minusvalías) o bien por decisión personal, elegían una formación más acorde a sus necesidades (Martínez-Caro, 2008).

La razón de medir la satisfacción de los estudiantes se fundamenta en el hecho de que son ellos el eje nuclear y la garantía de la existencia y mantenimiento de las organizaciones educativas. Los estudiantes como destinatarios de la educación son los que mejor pueden valorarla y, aunque puedan presentar una visión parcial y subjetiva, su opinión proporciona un referente que debe tomarse en cuenta.

Medir la satisfacción del alumno tiene sentido siempre que vaya acompañada de acciones que promuevan la mejora y la innovación; es por ello que medir dicho constructo de una manera consistente, permanente y adecuada, orientaría a la toma de decisiones correctas intentando aprovechar las oportunidades de mejora. En el presente estudio consideramos interesante conocer cuáles son las variables predictivas que pueden estar incidiendo en la satisfacción de los estudiantes, como indicadores de la calidad de la actividad docente llevada a cabo en la Universidad Nacional de Educación a Distancia. Con tal motivo, el objetivo es establecer cuáles son las variables que mayor impacto tienen en la satisfacción del estudiante y, por tanto, cuáles son los aspectos que se deberían tener en cuenta con la finalidad de alcanzar la excelencia en el ámbito universitario.

La estructura del artículo presenta en primer lugar el análisis de los factores que influyen en la satisfacción del estudiante, se plantean las hipótesis y se finaliza con los resultados y las conclusiones del estudio. 


\section{MÉTODO}

El alcance metodológico comprende dos etapas: la primera tiene que ver con la revisión bibliográfica de trabajos basados en la satisfacción de las instituciones de educación superior; y la segunda se refiere a la praxis que nos lleva a medir el nivel de satisfacción estudiantil, la cual pasó por los procesos validación y fiabilidad del instrumento usado para la medición, así como la identificación de las dimensiones subyacentes a la satisfacción estudiantil y el análisis de los resultados para el establecimiento de los criterios de la metodología propuesta.

Se ha llevado a cabo un estudio descriptivo e inferencial de carácter transversal que emplea una muestra no probabilística de sujetos voluntarios.

\section{Participantes}

La muestra del estudio está formada por un total de 60 estudiantes (39 mujeres y 21 hombres) con edades comprendidas entre 24 y 50 años del último curso de carrera de la Universidad Nacional de Educación a Distancia. La tabla 1 recoge una descripción más exhaustiva de las variables sociodemográficas objeto de estudio.

Tabla 1. Datos académicos y personales de la muestra

\begin{tabular}{|c|c|c|c|c|}
\hline Datos & Categorías & Dimensiones & Frecuencias & Porcentajes \\
\hline \multirow{4}{*}{ Datos académicos } & \multirow{4}{*}{ ÁMBITO } & Artes y humanidades & 11 & 18.3 \\
\hline & & Ciencias & 7 & 11.7 \\
\hline & & Ciencias de la salud & 8 & $13 \cdot 3$ \\
\hline & & Ciencias sociales y jurídicas & 34 & 56.7 \\
\hline \multirow{5}{*}{ Datos personales } & \multirow{2}{*}{ SEXO } & Hombre & 21 & 35.0 \\
\hline & & Mujer & 39 & 65.0 \\
\hline & \multirow{3}{*}{ EDAD } & Menos de 30 & 20 & 33.3 \\
\hline & & $31-40$ & 23 & 28.3 \\
\hline & & 41 a 50 & 17 & 16.0 \\
\hline
\end{tabular}

\section{Instrumento}

Los instrumentos mayoritariamente utilizados para medir la satisfacción con la experiencia universitaria han sido los cuestionarios y escalas. Entre ellos está el College Student Satisfaction Questionnaire (CSSQ) muy utilizado y desarrollado por Betz, Menne, Starr y Klingensmith (1971) y la Escala de Satisfacción Académica construida por Lent, Singley, Sheu, Janet, Schmidt y Schmidt (2007). Por su parte, Gento Palacios y García Vivas (2003) elaboran el cuestionario SEUE para conocer la 
satisfacción de los estudiantes universitarios con su educación. En el mismo contexto venezolano, Mejías y Martínez (2009) desarrollaron un instrumento para medir la Satisfacción Estudiantil en Educación Superior (SEU).

En el presente estudio se utilizó el Cuestionario de Satisfacción con la Experiencia Universitaria (CSEU) de Kember y Leung (2005) validado en su versión española por González-Peiteado, Pino-Juste y Penado-Abilleira (2016) con resultados muy satisfactorios en relación a las garantías de calidad métrica (viabilidad, validez y fiabilidad). Dicho instrumento ha requerido un procedimiento de adaptación y traducción, empleando el proceso de retro-traducción, siguiendo alguno de los trabajos publicados (Muñiz y Bartram, 2007) para garantizar una equivalencia completa entre el test original y el test traducido. Se obtuvo evidencia a favor de una estructura de cuatro dimensiones que en líneas generales valoran capacidades, enseñanza y ambiente de aprendizaje y que se denominan capacidad, docencia, contenidos y comunicación. Consta de 26 ítems y se responde en un formato de cinco alternativas de respuesta, sin encontrar un buen ajuste al intentar replicar una estructura de más dimensiones. El CSEU en su versión original consta de 13 escalas compuestas por dos ítems cada una, registrándose índices moderados pero aceptables de fiabilidad. Sin embargo, en nuestro estudio se ha reducido de 13 factores a 4 dimensiones mejorando considerablemente los resultados psicométricos.

\section{Análisis de datos}

El análisis de datos se llevó a cabo usando el paquete estadístico SPSS (V.20.o.). En primer lugar se efectuaron estudios de estructura y consistencia interna realizando un análisis factorial exploratorio y coeficiente Alfa de Cronbach. En segundo lugar, se llevó a cabo un análisis descriptivo de los datos considerando medias y desviaciones típicas. A continuación, se emplea la prueba de Kolmogorov Smirnov con el propósito de decidir de un modo riguroso si la muestra de la que se dispone procede o no de una distribución normal y, seguidamente, se realizaron Análisis de Varianza (pruebas post-hoc en caso de resultar significativos), Pruebas $T$ de comparación de medias independientes con un nivel de significación de $p<.05 \mathrm{y}$ pruebas Chi-Cuadrado para comprobar si las puntuaciones en las dimensiones de la satisfacción eran diferentes atendiendo a las variables sociodemográficas. También se calcularon los Tamaños del Efecto -TE, $d$ (diferencia media tipificada, Hedges y Olkin, 1985) entre las mujeres y los varones. Posteriormente se analizó la relación entre los factores de satisfacción mediante los coeficientes de correlación de Pearson.

\section{RESULTADOS}

Para conocer la fiabilidad del instrumento se realiza la prueba general del modelo, así como para cada una de las dimensiones. El alfa de Cronbach en la 
presente investigación fue muy satisfactoria $(\alpha=0.96)$. En la tabla 2 se exponen las dimensiones y su fiabilidad media que oscilan entre 0.80 y 0.94.

Tabla 2. Puntuaciones medias, desviación típica, alpha Cronbach

\begin{tabular}{|l|c|c|c|c|}
\hline \multicolumn{1}{|c|}{ Factores } & $\begin{array}{c}N^{0} \text { de } \\
\text { elementos }\end{array}$ & Alpha Cronbach & Media & $\begin{array}{c}\text { Desviación } \\
\text { Típica }\end{array}$ \\
\hline Capacidades & 9 & .94 & 33.70 & 6.80 \\
\hline Personal docente & 8 & .96 & 24.73 & 8.05 \\
\hline Contenidos & 5 & .94 & 17.43 & 4.79 \\
\hline Comunicación & 4 & .80 & 13.23 & 4.03 \\
\hline
\end{tabular}

Seguidamente se llevó a cabo un análisis factorial exploratorio mediante extracción por componentes principales y rotación oblicua, ya que se partía de que los posibles factores a extraer estarían relacionados entre sí. El coeficiente KMO encontrado fue de .90 y la prueba de esfericidad de Bartlett obtuvo un de $\mathrm{X}^{2}=$ 1661.92, significativo para 320 grados de libertad $(p \leq .0001)$. Estos datos avalan los supuestos necesarios para llevar a cabo el análisis factorial (González-Peiteado, Pino-Juste, y Penado-Abilleira, 2016) con el propósito de examinar el grado en que los indicadores definidos evalúan adecuadamente el concepto que se quiere medir "la satisfacción del alumnado".

Se obtuvo una solución de cuatro dimensiones que ofrecía una mejor interpretación teórica de los factores, reduciéndose el número de componentes del modelo teórico de referencia explicando de forma conjunta el $80.24 \%$ de la varianza y con un gran peso del primer factor cuyo porcentaje de varianza explicada es claramente mayor que el de las otras tres dimensiones como se recoge en la tabla 3.

Tabla 3. Dimensiones de Satisfacción

\begin{tabular}{|c|c|c|c|c|}
\hline Elementos & $\begin{array}{c}\text { Factor 1 } \\
\text { Capacidad }\end{array}$ & $\begin{array}{c}\text { Factor 2 } \\
\text { Docencia }\end{array}$ & $\begin{array}{c}\text { Factor 3 } \\
\text { Contenidos }\end{array}$ & $\begin{array}{c}\text { Factor 4 } \\
\text { Comunicación }\end{array}$ \\
\hline 1. & .61 & & & \\
\hline 2. & .47 & & & \\
\hline 3. & .83 & & & \\
\hline 4. & .67 & & & \\
\hline 5. & .74 & & & \\
\hline 6. & .73 & & & \\
\hline 7. & .70 & & & \\
\hline 8. & .78 & & & \\
\hline 9. & .73 & & & \\
\hline
\end{tabular}




\begin{tabular}{|c|c|c|c|c|}
\hline Elementos & $\begin{array}{c}\text { Factor } 1 \\
\text { Capacidad }\end{array}$ & $\begin{array}{l}\text { Factor } 2 \\
\text { Docencia }\end{array}$ & $\begin{array}{c}\text { Factor } 3 \\
\text { Contenidos }\end{array}$ & $\begin{array}{c}\text { Factor } 4 \\
\text { Comunicación }\end{array}$ \\
\hline 10. & & .74 & & \\
\hline 11. & & .66 & & \\
\hline 12. & & .83 & & \\
\hline 13. & & .80 & & \\
\hline 14. & & .85 & & \\
\hline 15. & & .86 & & \\
\hline 16. & & .82 & & \\
\hline 17. & & .86 & & \\
\hline 18. & & & .50 & \\
\hline 19. & & & .68 & \\
\hline 20. & & & .76 & \\
\hline 21. & & & .67 & \\
\hline 22. & & & .66 & \\
\hline 23. & & & & .65 \\
\hline 24. & & & & .83 \\
\hline 25. & & & & .78 \\
\hline 26. & & & & .53 \\
\hline Varianza & 14.61 & 3.68 & 1.42 & 1.03 \\
\hline \% Var & 56.21 & 14.55 & 5.49 & 3.99 \\
\hline $\begin{array}{c}\% \\
\text { Acumulada }\end{array}$ & 56.21 & 70.76 & 76.25 & 80.24 \\
\hline
\end{tabular}

En lo referente a la validez de constructo y para completar este análisis preliminar de datos, se llevó a cabo una matriz de correlaciones de Pearson entre los factores de la escala. En la tabla 4 se presenta la matriz de correlaciones de los factores encontrados, evidenciándose muy aceptables índices de correlación en las cuatro dimensiones que van desde 0.58 hasta 0.82 siguiendo un patrón de correlaciones moderadas y altas y estadísticas significativas a nivel de o.o1.

Tabla 4. Correlaciones entre factores

\begin{tabular}{|l|c|c|c|c|}
\hline \multicolumn{1}{|c|}{ Factores } & Capacidad & Docencia & Contenidos & Comunicación \\
\hline Capacidad & - & & & \\
\hline Docencia & $.58^{* *}$ & - & & \\
\hline Contenidos & $.82^{* *}$ & $.64^{* *}$ & - & \\
\hline Comunicación & $.68^{* *}$ & $.65^{* *}$ & $.78^{* *}$ & - \\
\hline
\end{tabular}

** La correlación es significativa al nivel o,o1 (bilateral). 
Por lo que respecta al análisis descriptivo de los ítems podemos comprobar las medidas de tendencia central en la tabla 5. Como se puede comprobar, las medias en cada una de los ítems son relativamente altas destacando negativamente la no existencia de relaciones entre el personal docente y los estudiantes y positivamente el compromiso de los estudiantes en asumir la responsabilidad sobre su propio aprendizaje.

Tabla 5. Análisis descriptivo

\begin{tabular}{|c|c|c|c|c|}
\hline Factores & Ítems & Media & DT & Moda \\
\hline \multirow{9}{*}{ 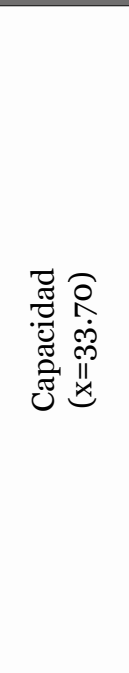 } & $\begin{array}{l}\text { A través del Grado cursado he desarrollado mi capacidad de } \\
\text { hacer juicios de valor sobre perspectivas opuestas. }\end{array}$ & 3.55 & 0.93 & 4 \\
\hline & Estoy más dispuesto a considerar distintos puntos de vista. & 3.73 & 0.97 & 4 \\
\hline & $\begin{array}{l}\text { Frente a problemas difíciles, a menudo puedo encontrar } \\
\text { nuevas formas de resolverlos. }\end{array}$ & 3.50 & 1.05 & 4 \\
\hline & $\begin{array}{l}\text { Durante mi período universitario, he aprendido a adaptarme } \\
\text { mejor. }\end{array}$ & 3.70 & 0.94 & 4 \\
\hline & $\begin{array}{l}\text { Siento que puedo asumir la responsabilidad de mi propio } \\
\text { aprendizaje. }\end{array}$ & 4.02 & 0.83 & 4 \\
\hline & $\begin{array}{l}\text { Confío más en mis habilidades para afrontar nuevos } \\
\text { aprendizajes. }\end{array}$ & 3.98 & 0.87 & 4 \\
\hline & Estoy más dispuesto a cambiar y a aceptar nuevas ideas. & 3.68 & 0.85 & 4 \\
\hline & $\begin{array}{l}\text { He mejorado mi capacidad de utilizar el conocimiento para } \\
\text { resolver problemas de forma sistemática. }\end{array}$ & 3.68 & 0.95 & 4 \\
\hline & $\begin{array}{l}\text { Soy capaz de interrelacionar la información y las ideas de } \\
\text { diferentes temas para resolver problemas. }\end{array}$ & 3.83 & 0.88 & 4 \\
\hline \multirow{8}{*}{ 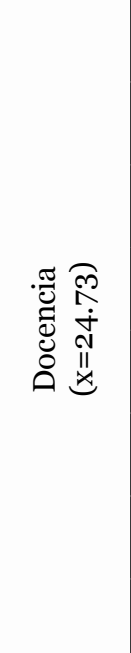 } & $\begin{array}{l}\text { Nuestro personal docente utiliza una variedad de métodos de } \\
\text { enseñanza. }\end{array}$ & 3.03 & 1.19 & 2 \\
\hline & $\begin{array}{l}\text { Los estudiantes tuvieron la oportunidad de participar en la } \\
\text { clase. }\end{array}$ & 3.15 & 1.12 & 3 \\
\hline & $\begin{array}{l}\text { El profesorado se esfuerza por hacernos comprender el } \\
\text { material del curso. }\end{array}$ & 3.18 & 1.18 & 3 \\
\hline & $\begin{array}{l}\text { Los profesores diseñan las clases con el objetivo de que los } \\
\text { estudiantes logren la comprensión de los contenidos del curso. }\end{array}$ & 3.23 & 1.08 & 3 \\
\hline & $\begin{array}{l}\text { Cuando tuve problemas con el material del curso, los } \\
\text { profesores estaban disponibles para ayudar. }\end{array}$ & 3.17 & 1.13 & 3 \\
\hline & $\begin{array}{l}\text { El personal docente fue útil cuando tuve problemas para } \\
\text { comprender el material del curso. }\end{array}$ & 3.18 & 1.14 & 4 \\
\hline & $\begin{array}{l}\text { Existe una estrecha relación entre el personal docente y los } \\
\text { estudiantes. }\end{array}$ & 2.77 & 1.22 & 2 \\
\hline & $\begin{array}{l}\text { Hubo una buena comunicación entre el personal docente y los } \\
\text { estudiantes. }\end{array}$ & 3.02 & 1.12 & 3 \\
\hline
\end{tabular}




\begin{tabular}{|c|c|c|c|c|}
\hline Factores & Ítems & Media & DT & Moda \\
\hline \multirow{5}{*}{ 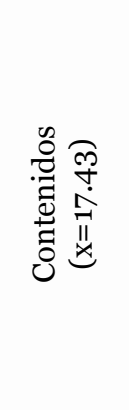 } & $\begin{array}{l}\text { En esta carrera me animaron a examinar cuestiones o } \\
\text { problemas existentes de otro modo. }\end{array}$ & $3 \cdot 37$ & 1.06 & 4 \\
\hline & $\begin{array}{l}\text { Creo que los contenidos han mejorado mi rendimiento en el } \\
\text { trabajo. }\end{array}$ & 3.58 & 1.10 & 4 \\
\hline & $\begin{array}{l}\text { El contenido de las asignaturas de Grado responderá bien a } \\
\text { las necesidades de mi carrera. }\end{array}$ & 3.42 & 1.09 & 4 \\
\hline & $\begin{array}{l}\text { Adquirí un montón de conocimientos y habilidades útiles en } \\
\text { la carrera. }\end{array}$ & $3 \cdot 50$ & 1.02 & 3 \\
\hline & $\begin{array}{l}\text { He desarrollado una buena comprensión de los principales } \\
\text { conceptos en mi área. }\end{array}$ & 3.67 & 1.03 & 4 \\
\hline \multirow{4}{*}{ 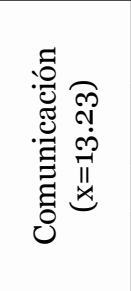 } & $\begin{array}{l}\text { En esta carrera he desarrollado mi habilidad para comunicarme } \\
\text { efectivamente con los demás. }\end{array}$ & 3.27 & 1.10 & 4 \\
\hline & $\begin{array}{l}\text { Durante mi etapa universitaria he mejorado mis habilidades } \\
\text { de presentación. }\end{array}$ & 3.22 & 1.14 & 4 \\
\hline & Aprendí cómo ser un miembro efectivo de un equipo o grupo. & 3.03 & 1.14 & 3 \\
\hline & $\begin{array}{l}\text { Estoy seguro de que puedo tratar con una amplia variedad de } \\
\text { personas. }\end{array}$ & 3.72 & 1.19 & 4 \\
\hline
\end{tabular}

Con la finalidad de conocer el tipo de análisis a realizar, se han comprobado los supuestos de normalidad. A continuación en la tabla 6 se recogen los valores del análisis de la prueba de Kolmogorov-Smirnov, relativa a la normalidad de la muestra, obteniéndose que sigue una distribución normal ( $p>$.05). En consecuencia se realizaran pruebas paramétricas.

Tabla 6. Prueba Kolmogorov-Smirnov para una muestra

\begin{tabular}{|l|l|c|}
\hline & & Total Ítems \\
\hline \multirow{2}{*}{ Parámetros normales } & Media & 3.42 \\
& Desviación típica & .78 \\
\hline \multirow{3}{*}{ Diferencias más extremas } & Absoluta & .06 \\
& Positiva & .05 \\
& Negativa & -.06 \\
\hline Z de Kolmogorov-Smirnov & & .47 \\
\hline Sig.asintót. (bilateral) & & .98 \\
\hline
\end{tabular}

La distribución de contraste es la normal. Se ha calculado a partir de los datos

El análisis post-hoc indicó diferencias entre los estudiantes en función de su ámbito de formación en el factor comunicación. Los estudiantes de Ciencias son los que puntúan más alto presentando diferencias significativas con el ámbito de 
“Artes y Humanidades" y de "Ciencias de la Salud". En la tabla 7 se presentan las puntuaciones registradas en cada una de las cuatro dimensiones.

Tabla 7. ANOVA y Bonferroni en función del ámbito de estudio

\begin{tabular}{|c|c|c|c|c|c|c|c|c|}
\hline FACTORES & Formación & Media & DT & $\mathrm{t}$ & Sig. & Bon & ferroni Sig. & \\
\hline \multirow{12}{*}{ Comunicación } & \multirow{12}{*}{$\begin{array}{l}\text { Artes y } \\
\text { humanidades } \\
\text { Ciencias } \\
\text { Ciencias de la } \\
\text { salud } \\
\text { CC sociales/ } \\
\text { jurídicas }\end{array}$} & \multirow{12}{*}{$\begin{array}{c}16,33 \\
16,43 \\
14,66 \\
13.15\end{array}$} & \multirow{12}{*}{$\begin{array}{l}1,46 \\
1,41 \\
2,50 \\
3.92\end{array}$} & \multirow{12}{*}{3.43} & \multirow{12}{*}{.023} & \multirow{3}{*}{$\begin{array}{l}\text { Artes } \\
\text { Humanidades }\end{array}$} & Ciencias & .04 \\
\hline & & & & & & & $\begin{array}{l}\text { Ciencias de la } \\
\text { salud }\end{array}$ & 1.00 \\
\hline & & & & & & & $\begin{array}{l}\text { CC sociales/ } \\
\text { jurídicas }\end{array}$ & 1.00 \\
\hline & & & & & & \multirow{3}{*}{ Ciencias } & $\begin{array}{l}\text { Artes } \\
\text { Humanidades }\end{array}$ & .04 \\
\hline & & & & & & & $\begin{array}{l}\text { Ciencias de la } \\
\text { salud }\end{array}$ & .03 \\
\hline & & & & & & & $\begin{array}{l}\text { CC sociales/ } \\
\text { jurídicas }\end{array}$ & .13 \\
\hline & & & & & & \multirow{3}{*}{$\begin{array}{l}\text { Ciencias de la } \\
\text { salud }\end{array}$} & $\begin{array}{l}\text { Artes } \\
\text { Humanidades }\end{array}$ & 1.00 \\
\hline & & & & & & & Ciencias & .03 \\
\hline & & & & & & & $\begin{array}{l}\text { CC sociales/ } \\
\text { jurídicas }\end{array}$ & 1.00 \\
\hline & & & & & & \multirow{3}{*}{$\begin{array}{l}\text { CC sociales/ } \\
\text { jurídicas }\end{array}$} & $\begin{array}{l}\text { Artes y } \\
\text { humanidades }\end{array}$ & 1.00 \\
\hline & & & & & & & Ciencias & .13 \\
\hline & & & & & & & $\begin{array}{l}\text { Ciencias de la } \\
\text { salud }\end{array}$ & 1.00 \\
\hline
\end{tabular}

Con relación a la edad, se encontró una diferencia significativa en los factores "contenidos" y "comunicación" que indica que el tramo de edad comprendido entre 41 y 50 años están más satisfechos con los contenidos recibidos y los menores de 30 son los que reflejan una mayor insatisfacción. Resultados similares arroja el estudio del factor "comunicación". En la tabla 8 se muestran las estimaciones registradas en cada una de las cuatro escalas en función de la edad de los estudiantes participantes del estudio. 
Tabla 8. ANOVA y Bonferroni en función de la edad

\begin{tabular}{|c|c|c|c|c|c|c|c|c|}
\hline FACTORES & Edad & Media & DT & F & Sig. & \multicolumn{3}{|c|}{ Bonferroni Sig. } \\
\hline \multirow{6}{*}{ Contenidos } & \multirow{6}{*}{$\begin{array}{l}\text { Menos 30: } \\
\text { 31-40: } \\
\text { 41-50: }\end{array}$} & \multirow{6}{*}{$\begin{array}{l}14.70 \\
18.00 \\
19.88\end{array}$} & \multirow{6}{*}{$\begin{array}{l}4.73 \\
5.04 \\
2.60\end{array}$} & \multirow{6}{*}{6.74} & \multirow{6}{*}{0,00} & Menos 30 & $31-40$ & .04 \\
\hline & & & & & & & $41-50$ & .00 \\
\hline & & & & & & $31-40$ & Menos 30 & .04 \\
\hline & & & & & & & $41-50$ & .55 \\
\hline & & & & & & $41-50$ & Menos 30 & .00 \\
\hline & & & & & & & $31-40$ & .55 \\
\hline \multirow{6}{*}{ Comunicación } & \multirow{6}{*}{$\begin{array}{l}\text { Menos 30: } \\
\text { 31- 40: } \\
\text { 41-50: }\end{array}$} & \multirow{6}{*}{$\begin{array}{l}11.40 \\
13.43 \\
15.11\end{array}$} & \multirow{6}{*}{$\begin{array}{l}4.22 \\
4.15 \\
2.66\end{array}$} & \multirow{6}{*}{4.40} & \multirow{6}{*}{0,01} & Menos 30 & $31-40$ & .26 \\
\hline & & & & & & & $41-50$ & .01 \\
\hline & & & & & & $31-40$ & Menos 30 & .26 \\
\hline & & & & & & & $41-50$ & .52 \\
\hline & & & & & & $41-50$ & Menos 30 & .01 \\
\hline & & & & & & & $31-40$ & .52 \\
\hline
\end{tabular}

El estadístico $\mathrm{t}$ de Student para el contraste de medias de muestras independientes revela que la prueba de Levene no es significativa, por lo que asumimos la homogeneidad de varianzas sosteniendo que no hay asociación entre el sexo y los factores estudiados. En consecuencia, en la tabla 9 se observa que hombres y mujeres no difieren significativamente en la satisfacción con la experiencia universitaria en los factores estudiados. Sin embargo, debido a que una diferencia no estadísticamente significativa puede ser grande e importante en determinadas situaciones, hemos realizado el cálculo complementario del tamaño del efecto que nos informa de manera más precisa la diferencia entre dos medias. Dichos datos corroboran que la diferencia encontrada es pequeña en todos los factores a excepción del factor comunicación con un valor significativo $\mathrm{de} d=0.78$, siendo las mujeres las que presentan valores más altos.

Tabla 9. Prueba t de Student para muestras independientes y tamaño del efecto

\begin{tabular}{|c|c|c|c|c|c|c|}
\hline Factores & Sexo & Media & DT & $\mathrm{t}$ & Sig. & $\mathrm{TE}$ \\
\hline \multirow[t]{2}{*}{ Capacidad } & Hombre & 34.52 & 6.86 & \multirow{2}{*}{.70} & \multirow{2}{*}{.472} & \multirow{2}{*}{0.19} \\
\hline & Mujer & 33.23 & 6.78 & & & \\
\hline \multirow[t]{2}{*}{ Docencia } & Hombre & 26.24 & 7.87 & \multirow{2}{*}{1.06} & \multirow{2}{*}{.992} & \multirow{2}{*}{0.30} \\
\hline & Mujer & 23.92 & 8.12 & & & \\
\hline \multirow[t]{2}{*}{ Contenidos } & Hombre & 17.66 & 5.43 & \multirow{2}{*}{.27} & \multirow{2}{*}{.145} & \multirow{2}{*}{0.07} \\
\hline & Mujer & $17 \cdot 31$ & 4.47 & & & \\
\hline \multirow[t]{2}{*}{ Comunicación } & Hombre & 12.52 & 4.74 & \multirow{2}{*}{-.99} & \multirow{2}{*}{.065} & \multirow{2}{*}{0.78} \\
\hline & Mujer & 13.61 & 3.60 & & & \\
\hline
\end{tabular}




\section{DISCUSIÓN Y CONCLUSIONES}

Se ha utilizado en algunos estudios el Cuestionario de Satisfacción con la Experiencia Universitaria (CSEU) de Kember y Leung (2005) para evaluar diferentes estudios de grado, donde los resultados mostraron, por un lado, que la calidad del entorno de enseñanza y aprendizaje afecta de manera significativa al desarrollo de las competencias generales; por otro lado, durante un periodo de cinco años, sirvieron para llevar a cabo iniciativas de mejora de la calidad de la enseñanza (Gómez, Roca y Guerrero, 2014).

Los resultados de este estudio muestran el uso apropiado de la clasificación conceptual utilizada por el instrumento de medición utilizado y la evidencia empírica obtenida, la cual apoya dicha aseveración. De hecho, los resultados de las correlaciones entre los factores muestran la mutua influencia que tienen los factores entre sí, apoyando los argumentos teóricos presentados sobre la relación existente entre las necesidades intrínsecas de competencia, autonomía y correspondencia y la influencia del ambiente en la satisfacción de las mismas.

A través del estudio estadístico de los ítems hemos podido comprobar que la mayor parte de los estudiantes se sienten satisfechos con su vida universitaria manifestando un alto grado de bienestar con las diferentes variables analizadas. Los ítems peor valorados son los que integran el factor docencia, en concreto los que hacen referencia a la metodología docente y las interacciones docenteestudiante. En consecuencia, sería apropiado potenciar el uso de metodologías que optimicen la comunicación así como la influencia que la figura del profesor tiene sobre la satisfacción del estudiante con el propósito de elevar la calidad educativa y la satisfacción de los estudiantes con la experiencia universitaria. Sin embargo, los ítems pertenecientes a las dimensiones capacidad, contenidos y comunicación registran una alta valoración.

Si tenemos en cuenta los resultados del estudio inferencial, comprobamos la existencia de diferencias de opinión entre los estudiantes en función de su ámbito de formación de modo que los estudiantes de Ciencias son los que puntúan más alto presentando diferencias con el ámbito de Artes y Humanidades y de Ciencias de la Salud, pero no con Ciencias Sociales/Jurídicas.

Similares conclusiones se derivan del estudio de Tejedor et al. (1999) sobre la satisfacción del alumnado universitario, revelando que los más satisfechos son los alumnos de la especialidad de Biomédicas, seguido de los de Letras; a continuación, Ciencias y, por último, Ciencias Sociales y Económico-Jurídicas.

En relación con este último ámbito, el estudio de Blázquez et al. (2013) utiliza varios indicadores para medir la calidad de vida universitaria de los estudiantes de Ciencias Empresariales o de Administración y Dirección de Empresas de la Facultad de Ciencias Sociales de Talavera de la Reina, llegando a la conclusión de que la calidad de vida universitaria está determinada de una forma muy parecida tanto por la satisfacción con los aspectos docentes como por la satisfacción con los aspectos 
sociales. Los resultados muestran que la satisfacción global del alumno universitario repercute en su calidad de vida global y en su identificación con la institución. Este es un aspecto considerado importante para las universidades que tratan de atraer un mayor número de alumnado, valorando a este como un canal de comunicación muy importante.

En lo que se refiere a la variable edad, en nuestro estudio nos encontramos que existen diferencias de opinión con relación a la edad en las dimensiones "contenidos" y "comunicación" de modo que el tramo de edad comprendido entre 41 y 50 años están más satisfechos con los contenidos y comunicación y los menores de 30 son los que reflejan una mayor insatisfacción.

Estudios donde se ha analizado la satisfacción de los estudiantes con sus experiencias universitarias académicas como Clemes, Gan y Tzu-Hui (2008) sugieren que la percepción de las construcciones de los estudiantes se ven influidas principalmente por su origen étnico y el año de estudio y no por la variable sexo ni edad.

Al considerar la variable sexo hemos comprobado que no existen indicios estadísticos para pensar que el grado de satisfacción sea diferente entre hombres y mujeres a excepción de la dimensión "comunicación" que una vez realizada la prueba del tamaño del efecto arroja un valor significativo (o.78), manifestando una mayor puntuación las mujeres. Resultado que coincide, en gran parte, con el estudio llevado a cabo por Tejedor et al. (1999) donde no se manifiestan diferencias significativas en función de la variable sexo.

Sin embargo, en el caso de Ceyhan Aldemir y Yaprak Gülcan (2004), que tienen como objetivo determinar el nivel y los factores de satisfacción de los estudiantes universitarios con las instituciones, los resultados muestran que, al menos para algunos estudiantes universitarios turcos, la calidad de la educación, los profesores, los libros de texto, ser mujer y recibir información previa son importantes factores de satisfacción universitaria. En este caso podría ser importante analizar la influencia del factor cultural y del entorno.

En otros estudios se definen otras variables importantes. Por ejemplo, Mainardes, Alves y Raposo (2013) evalúan la satisfacción de los estudiantes en las universidades públicas portuguesas concluyendo que el nivel de exigencia, las expectativas laborales, el nivel de autorrealización, el clima de la universidad y las clases motivadoras son dimensiones que inciden en la satisfacción de los estudiantes.

Las dimensiones consideradas en este estudio proporcionan un punto de partida para poder generar un plan estratégico que lleve a optimizar la experiencia del estudiante en la universidad, con el propósito de elevar el desempeño docente y la calidad institucional en un intento de mejorar satisfacción del alumno.

Entre las limitaciones del estudio podemos destacar las derivadas de medir la satisfacción con la vida universitaria exclusivamente con una escala y también sería interesante ampliar la muestra con estudiantes de otras universidades así 
como vincular la satisfacción con la vida universitaria con otros constructos como rendimiento académico y motivación hacia el estudio.

\section{REFERENCIAS BIBLIOGRÁFICAS}

Allen, M., Omori, K., Burrell, N., Mabry, E., y Timmerman, E. (2013). Satisfaction with distance education. In M. G. Moore (Ed.), Handbook of distance education. 3ra. ed. (143-154). Nueva York: Routledge.

Betz, E. L., Menne, J. W., Starr, A. M., y Klingensmith, J. E. A. (1971). Dimensional Analysis of College Student Satisfaction. Measurement and Evaluation in Guidance, 42, 99-106.

Blázquez Resino, J. C., Chamizo González, J., Cano Montero, E. I., y Gutiérrez Broncano, S. (2013). Calidad de vida universitaria: Identificación de los principales indicadores de satisfacción estudiantil. Revista de Educación, 362. 458-484 doi: 10.4438/1988-592X-RE2013-362-238

Ceyhan, A., y Yaprak, G. (2004). Student Satisfaction in Higher Education: A Turkish Case. Higher Education Management and Policy, 16(2), 109-122 doi: $10.1787 / 17269822$

Chow, H. P. H. (2005). Life Satisfaction among University Students in a Canadian Prairie City: A Multivariate Analysis. Social Indicators Research, 70(2), 139150.

El Hassan, K. (2011). Quality of College Life (QCL): Validation of a Measure of Student Well-Being in the Middle East. The International Journal of Educational and Psychological Assessment, 8(1), 1222.

Fernández-Abascal, E., García Rodríguez, B., Jiménez Sánchez, M. D., y Domínguez Sánchez, F. J. (2011). Psicología de la emoción. Madrid: Ramón Areces.

Fernández-Pascual, M. D., Ferrer-Casales, R., y Reig-Ferrer, A. (2013). Entornos virtuales: predicción de la satisfacción en contexto universitario. Píxel-Bit. Revista de Medios y Educación, 43, 167181. doi: http://dx.doi.org/10.12795/ pixelbit.2013.i43.12

Flores Alarcia, O. (2012). TIC y docencia universitaria: ¿cambian las metodologías docentes según el grado de presencialidad de las asignaturas? El caso de la Universidad de Lleida. Píxel-Bit. Revista de Medios y Educación, 41, 63-76.

García Aretio, L. (2001). La Educación a Distancia. De la Teoría a la Práctica. Barcelona: Ariel Educación.

García Aretio, L. (2016). Los inicios históricos de una compleja universidad pública a distancia: la UNED de España. RIED. Revista Iberoamericana de Educación a Distancia, 19(1), 9-21. doi: http://dx.doi. org/10.5944/ried.19.1.15057

Gento Palacios, S., y Vivas García, M. (2003). EL SEUE: Un Instrumento para Conocer la Satisfacción de los Estudiantes Universitarios con su Educación. Acción Pedagógica, 12(2), 16-27.

Gómez, L., Roca, C., y Guerrero, F. (2014). ¿Cómo perciben los estudiantes la adquisición de competencias? Análisis comparado: Teorías de la Comunicación en la Universidad Pompeu Fabra. Historia y Comunicación Social, 19, 313326. doi: http://dx.doi.org/10.5209/rev HICS.2014.v19.45030

González-Peiteado, M., Pino-Juste, M., y Penado-Abilleira, M. (2016). Valoración psicométrica de un cuestionario para medir la satisfacción con la experiencia universitaria. Revista Magis. En prensa.

Hedges, L. V., y Olkin, I. (1985). Statistical methods for meta-analysis. Orlando FL: Academic Press. 
Mainardes, E., Alves, A., y Raposo, M. (2013). Identifying stakeholders in a Portuguese university: a case study. Revista de Educación, 362, 429-457 doi: 10.4438/1988-592X-RE-2012-362-167

Joo, Y. J., Lim, K.Y., y Kim, E. K. (2011). Online university students' satisfaction and persistence: Examining perceived level of presence, usefulness and ease of use as predictors in a structural model. Computers \& Education, 57(2), 1654-1664. doi:10.1016/j.compedu.2011.02.008

Keengwe, J., Diteeyont, W., y LawsonBody, A. (2012). Student and Instructor satisfaction with e-learning tools in online learning environments. International Journal of Information and Comunication Technology Education, 8 (1), 76-86 doi:http://dx.doi.org/10.4018/ iicte.2012010108

Kember, D., y Leung, D. Y. P. (2005). The influence of active learning experiences on the development of graduate capabilities. Studies in Higher Education, 3O(2), 155170. doi: 10.1080/03075070500043127

Lee, S. J., Srinivasan, S., Trail, T., Lewis, D., y Lopez, S. (2011). Examining the relationship among student perception of support, course satisfaction, and learning outcomes in online learning. The Internet and Higher Education, 14(3), 158-163. doi: http://dx.doi.org/10.1016/i. iheduc.2011.04.001

Lent, R. W., Singley, D., Sheu, H., Janet B., Schmidt, A., y Schmidt, C. L. (2007). Relation of Social-Cognitive Factors to Academic Satisfaction in Engineering Student. Journal of Career Assessmen, 15, 87-97.

Martínez-Caro, E. (2008). E-learning: un análisis desde el punto de vista del alumno. RIED. Revista Iberoamericana de Educación a Distancia, 11(2), 151168. doi: http://dx.doi.org/10.5944 ried.2.11.948

Medrano, L. A., y Pérez E. (2010). Adaptación de la Escala de Satisfacción Académica a la Población Universitaria de Córdoba. SUMMA Psicológica UST, 7(2), 5-14.

Moore, J. C., y Shelton, K. (2014). The Sloan Consortium pillars and quality scorecard. In K. Shattuck (Ed.), Assuring quality in online education: Practices and processes at the teaching, resource, and program levels. Sterling, Virginia: Stylus Publishing, LLC.

Muñiz, J., y Bartram, D. (2007). Improving international tests and testing. European Psychologist, 12, 206-219.

Özkök, A., Walker, S. L., y Büyüköztürk, S. (2009). Reliability and validity of a Turkish version of the DELES. Learning Environments Research, 12, 175-190 doi: http://dx.doi.org/10.1007/s10984-0092060-0

Pérez Juste, R. (2000). La calidad de la educación. En R. Pérez, F. López, M. Peralta y P. Municio, Hacia una educación de calidad. Gestión, instrumentos y evaluación. Madrid: Narcea.

Sánchez-López, M. C., García-Sánchez, F. A., Martínez-Segura, M. J., y Mirete, A. (2012). Aproximación a la valoración que el alumnado hace de recursos online utilizados para la docencia universitaria. Píxel Bit. Revista de Medios y Educación, 4O, 35-45.

Sirgy, M. J., Grezeskowiak, S., y Rahtz, D. (2007). Quality of College Life (QCL) of Students: Developing and Validating a Measure of Well-Being. Social Indicators Research, 8o(2), 343-360.

Solinas, G., Masia, M. D., Maida, G., y Muresu, E. (2012). What Really Affects Student Satisfaction? An Assessment of Quality through a University-Wide Student Survey. Creative Education, 3(1), 37-40. doi: http://dx.doi.org/10.4236/ ce.2012.31006

Tejedor Tejedor, F. J., Martín Izard, J., García-Valcárcel, A., y Ausín Zorrilla, T. (1999). La satisfacción de los alumnos universitarios respecto a sus estudios. 
Revista de Investigación Educativa, 17(2), 407-412.

Váez, M., Kristenson, M., y Laflamme, L. (2004). Perceived Quality of Life and SelfRated Health among First-Year University Students. Social Indicators Research, 68(2), 221-234.

Yu, G., y Lee, D. (2008). A Model of Quality of College Life (QCL) of Students in
Korea. Social Indicators Research, 87(2), 269-285.

Zambrano Ramírez, J. (2016). Factores predictores de la satisfacción de estudiantes de cursos virtuales. RIED. Revista Iberoamericana de Educación a Distancia, 19(2), 217-235. doi: http:// dx.doi.org/10.5944/ried.19.2.15112

\section{PERFIL ACADÉMICO Y PROFESIONAL DE LAS AUTORAS}

Margarita González-Peiteado. Doctora en Ciencias de la Educación por la Universidad de Vigo. Licenciada en Ciencias de la Educación por la UNED. Diplomada en Formación del Profesorado de Educación General Básica. En la actualidad es profesora-tutora de la UNED en Pontevedra y Coordinadora del Aula UNED de Vigo. Sus líneas de investigación están centradas en la Formación Inicial del Profesorado y los Estilos Docentes. Ha participado en Congresos y publicado en revistas de reconocido prestigio nacional e internacional.

E-mail: margonzalez@pontevedra.uned.es

María Penado Abilleira. Doctora en Psicología por la Universidad Complutense de Madrid. Profesora tutora del Centro Asociado de la UNED en Pontevedra donde ejerce funciones de secretaria y coordinadora académica. Sus líneas de investigación se centran en el ámbito de la psicología básica, donde ha publicado artículos en revistas nacionales de prestigio.

E-mail: marabilleira@pontevedra.uned.es

\section{DIRECCIÓN DE LAS AUTORAS}

Universidad Nacional a Distancia. Centro Asociado de Pontevedra.

Rúa de Portugal, 1, 36004

Pontevedra. España

Margarita Pino-Juste. Doctora en Filosofía y Ciencias de la Educación por la Universidad de Santiago de Compostela. Profesora Titular del Departamento de Didáctica, Organización escolar y Métodos de Investigación de la Universidad de Vigo. Sus líneas de investigación se centran en el diseño y evaluación de programas educativos. Importantes colaboraciones en congresos y simposios internacionales, así como numerosas publicaciones en revistas del ámbito educativo y sanitario indizadas en prestigiosas bases de datos y con alto índice de impacto.

E-mail: mpino@uvigo.es 


\section{DIRECCIÓN DE LA AUTORA}

Facultad de Educación

Universidad de Vigo

Campus a Xunqueira, s/n 36005

Pontevedra. España

Fecha de recepción del artículo: 16/04/2016

Fecha de aceptación del artículo: 15/06/2016

\section{Como citar este artículo:}

González-Peiteado, M., Pino-Juste, M., y Penado-Abilleira, M. (2017). Estudio de la satisfacción percibida por los estudiantes de la UNED con su vida universitaria. RIED. Revista Iberoamericana de Educación a Distancia, 2O(1), pp. 243-260. doi: http://dx.doi.org/10.5944/ried.20.1.16377 